\title{
Nuclear features of infiltrating urothelial carcinoma are distinguished from low-grade noninvasive papillary urothelial carcinoma by image analysis
}

\author{
NORITAKE KOSUGE ${ }^{1}$, MASANAO SAIO ${ }^{1-3}$, HIROFUMI MATSUMOTO ${ }^{1,2}$, HAJIME AOYAMA ${ }^{1}$, \\ AKIKO MATSUZAKI $^{1}$ and NAOKI YOSHIMI ${ }^{1}$ \\ ${ }^{1}$ Department of Pathology and Oncology, Graduate School of Medicine, University of the Ryukyus; \\ ${ }^{2}$ Department of Pathology, University of The Ryukyus Hospital, Nishihara, Nakagami, Okinawa 903-0215; \\ ${ }^{3}$ Department of Laboratory Sciences, Gunma University School of Health Sciences, Maebashi, Gunma 371-8514, Japan
}

Received March 25, 2017; Accepted May 18, 2017

DOI: $10.3892 / 01.2017 .6474$

\begin{abstract}
Recent advances in computer technology have been made and image analysis (IA) has been introduced into pathological fields. The present study aimed to investigate the utility of IA for the evaluation of nuclear features and staining of immunohistochemistry (IHC) for Ki-67, p53 and GATA-binding protein 3 (GATA-3) in urothelial carcinoma tissue samples. A total of 49 cases of urothelial carcinoma tissue samples were obtained by transurethral resection of bladder tumors, which included 11 low-grade papillary urothelial carcinomas (LGPUCs), 1 non-invasive high-grade urothelial carcinoma and 37 infiltrating urothelial carcinomas (IUCs). Whole slide imaging (WSI) and IA were performed in Feulgen reaction and IHC-stained tissue samples. There was a significant difference in the average nuclear density, standard deviation (SD) of nuclear size and SD of nuclear minimum and maximum diameter between LGPUC and IUC, which is equivalent to the diagnostic features of IUC in nuclear variability, and hyperchromatic nuclei. In addition, the present study revealed that the SD of nuclear density was significantly different between the two groups. Regarding IA in IHC-stained tissue samples, Ki-67 was significantly overexpressed in IUC. Furthermore, the GATA-3 expression level in IUC samples with muscle invasion was significantly downregulated compared with that in non-muscle invasive tumors. The results of the present study suggest that IA in combination with WSI may be a beneficial tool for evaluating morphometric characteristics and performing semi-quantitative analysis of IHC.
\end{abstract}

Correspondence to: Dr Masanao Saio, Department of Laboratory Sciences, Gunma University School of Health Sciences, 39-22 3-chome, Showa-machi, Maebashi, Gunma 371-8514, Japan E-mail: saio@gunma-u.ac.jp

Key words: whole slide imaging, image analysis, urothelial carcinoma, nuclear morphology, Ki-67, p53, GATA-binding protein 3

\section{Introduction}

Digital pathology by whole slide imaging (WSI) has been utilized for education, diagnosis and research purposes (1). Recently, it was reported that the concordance rate of diagnosis by light microscopy and WSI was $92.4 \%$; therefore, the quality of WSI has been improved for the utilization of routine pathological diagnosis (2). In addition, image analysis (IA) by digital pathology has been used to perform quantification of liver fibrosis (3), nuclear morphological analysis of breast tumors (4) and other purposes, whereas digital pathological analysis for urothelial carcinoma has not been used in many previous studies. A previous study used pattern recognition algorithms for the diagnosis of urothelial carcinoma (5).

Nuclear findings are an important parameter in order to classify and diagnose urothelial tumors. For example, in the World Health Organization (WHO) Classification of Tumors of the Urinary Systems and Male Genital Organs 4th edition published in 2016 (6), the most infiltrating urothelial carcinoma (IUC) originated from noninvasive high-grade urothelial carcinoma (NIHGUC) (6), and the nuclear findings of IUC were described as 'striking nuclear pleomorphism with variably sized and shaped hyperchromatic nuclei' (6). Nuclear features of NIHGUC include size variation, irregular shape and apparent pleomorphic nuclei (7). Therefore, the nuclear findings between IUC and NIHGUC are similar. However, nuclear features of low-grade papillary urothelial carcinoma (LGPUC) include mild nuclear irregularity and evident pleomorphism (7). Therefore, it was suggested that LGPUC and IUC may be distinguished by nuclear irregularity and nuclear pleomorphism. However, to the best of our knowledge, there are no previous studies demonstrating that nuclear parameters may be useful to distinguish IUC from LGPUC through using IA of WSI samples. In addition, IA has been used to evaluate immunohistochemistry (IHC) (8); however, a number of studies still manually evaluate IHC $(9,10)$.

Therefore, the present study aimed to utilize IA for nuclear morphometric analysis and IHC evaluation of urothelial carcinoma tissue samples obtained by transurethral resection of bladder tumors (TUR-Bt). 


\section{Materials and methods}

Samples and ethical review. The tissue samples used in the present study had undergone a routine diagnostic process prior to submission of the present study's protocol to the Ethical Review Board of the University of the Ryukyus (Nakagami, Japan). All samples were initially obtained for pathological diagnosis. In the process of explaining to patients the use of sampling for pathological diagnosis, secondary usage for research was simultaneously explained by a clinician and written informed consent for secondary usage was obtained from the patients. Subsequently, the present study was initiated following approval from the Ethical Review Board. A total of 49 cases of urothelial carcinoma were obtained by TUR-Bt at the University of the Ryukyus Hospital between January 2011 and July 2015. For the present study, all tissue samples were reviewed by two pathologists (both from the Department of Pathology and Oncology, Graduate School of Medicine, University of the Ryukyus, Nishihara, Japan) independently, and depth of invasion and histological grade were evaluated, and classified based on the criteria of WHO classification and tumor-node-metastasis classification according to the Union for International Cancer Control 7th edition (11). Following individual evaluations, a consensus decision was made, and the consensus data was utilized in the present study. The data is summarized in Table I.

Hematoxylin and eosin $(H \& E)$, Feulgen reaction and $I H C$ staining. Buffered formalin-fixed paraffin embedded tissue samples (10\%) were cut into $3 \mu \mathrm{m}$ serial sections for H\&E staining, nuclear staining by Feulgen reaction and IHC staining. H\&E staining was performed using a routine protocol. In brief, deparaffinization and rehydration steps were performed using xylene for $3 \mathrm{~min}$ twice, 100, 90 and 70\% ethanol for $1 \mathrm{~min}$ each. Following rinsing in running water once and de-ionized water (DW) thrice, tissue samples were stained with hematoxylin solution for $15 \mathrm{~min}$ at room temperature $(\mathrm{R} / \mathrm{T})$, followed by washing with running water for $10 \mathrm{~min}$ and rinsing with distilled water thrice. After soaking in $80 \%$ ethanol for $3 \mathrm{~min}$, the samples were stained with eosin solution for $3 \mathrm{~min}$. at $\mathrm{R} / \mathrm{T}$. Subsequently, the dehydration step (100\% ethanol for $1 \mathrm{~min}$ 5 times) and penetration step (xylene for 4 min 4 times) were performed, and the samples were mounted for observation and analysis. For Feulgen reaction staining, deparaffinized tissue samples were pretreated with DW for $5 \mathrm{~min}$ at $60^{\circ} \mathrm{C}$. Subsequently, the tissue samples were treated with $1 \mathrm{~N} \mathrm{HCl}$ for $60 \mathrm{~min}$ at $60^{\circ} \mathrm{C}$. The tissue samples were washed once with Schiff's reagent (Muto Pure Chemicals, Bunkyo-ward, Tokyo, Japan) and treated for $15 \mathrm{~min}$ at R/T with Schiff's reagent. Subsequently, the tissue samples were treated three times with sulfuric acid solution (Muto Pure Chemicals) for $2 \mathrm{~min}$ at R/T. Following washing with running water for 5 min, dehydration and penetration steps were performed, and the samples were mounted for observation and analysis. For IHC staining, deparaffinization, rehydration and antigen retrieval steps were simultaneously performed using target retrieval solution (pH 9.0) with PT Link equipment (Agilent Technologies, Inc., Santa Clara, CA, USA) at $97^{\circ} \mathrm{C}$ for $20 \mathrm{~min}$. Subsequently, the tissue samples were placed on a DAKO Autostainer Link 48 (Agilent Technologies, Inc.) for staining. An EnVision ${ }^{\mathrm{TM}}$ FLEX
Table I. Clinicopathological parameters of the 49 patients with urothelial carcinoma.

\begin{tabular}{lc}
\hline Parameter & No. of cases $(\%)$ \\
\hline All cases & $49(100)$ \\
Age, years & \\
$<60$ & $12(24)$ \\
$\geq 60$ & $37(76)$ \\
Sex & \\
Male & $40(82)$ \\
Female & $9(18)$ \\
pT status & \\
pTa & $12(24)$ \\
pT1 & $16(33)$ \\
pT2 & $21(43)$ \\
Histological grade & \\
Infiltrating & $37(76)$ \\
High & $1(2)$ \\
Low & $11(22)$ \\
\hline
\end{tabular}

pT, pathological T factor; pTa, papillary tumor without invasion; pT1, tumor with submucosal invasion; pT2, tumor with muscular invasion.

High pH kit (K8000; Agilent Technologies, Inc.), containing $20 \mathrm{X}$ concentrated washing buffer, blocking reagent to block internal peroxidase activity, horseradish peroxidase labeled polymer conjugated secondary antibody, 3,3'-diaminobenzidine (DAB), buffer for DAB and hematoxylin solution, was used. In the autostainer, the following steps were performed: Following rinsing with $1 \mathrm{x}$ washing buffer, the samples were incubated with blocking reagent for $5 \mathrm{~min}$ at $\mathrm{R} / \mathrm{T}$. After washing with washing buffer, the slides were incubated with purified primary monoclonal antibodies against either Ki-67 (1:100 dilution; clone MIB-1; M7240), p53 (1:100 dilution; clone DO-7; M7001) (both from Agilent Technologies, Inc.) or GATA-binding protein 3 (GATA-3; 1:250 dilution; clone L50-823; ACR405A; Biocare Medical, LLC, Paheco, CA, USA) for $20 \mathrm{~min}$ at $\mathrm{R} / \mathrm{T}$. Following rinsing with washing buffer, the slides were washed with washing buffer for $5 \mathrm{~min}$ at $R / T$, visualization was performed following incubation with DAB diluted in buffer for DAB for $10 \mathrm{~min}$ at $\mathrm{R} / \mathrm{T}$. After rinsing with washing buffer, counterstaining was performed with hematoxylin solution for $5 \mathrm{~min}$ at R/T followed by a DW rinse, washing buffer for $5 \mathrm{~min}$ and a DW rinse. After this step, the slides were taken from the autostainer and then the dehydration step (100\% ethanol for $1 \mathrm{~min} 5$ times) and penetration step (xylene for 4 min 4 times) were performed, and the samples were mounted for observation for analysis.

WSI. WSI of all tissue samples was performed using a TOCO 240 Virtual slide scanner (Claro, Hirosaki, Aomori, Japan). For H\&E and Feulgen reaction stained tissue samples, a 40X objective lens was used and a 20X objective lens was used for the IHC tissue samples. The TOCO 240 specifications were as follows: Camera pixels, 1,360x1,240; size of the pixel, 
A

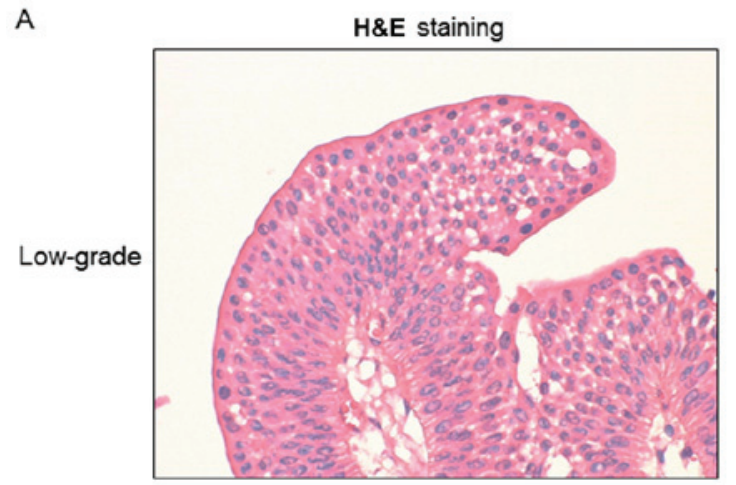

C

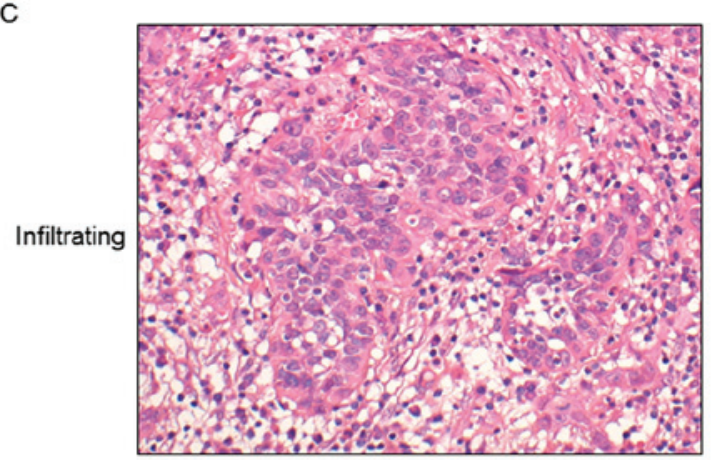

B

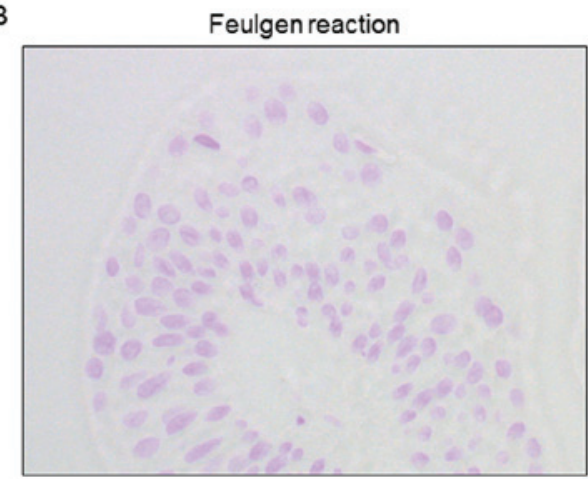

D

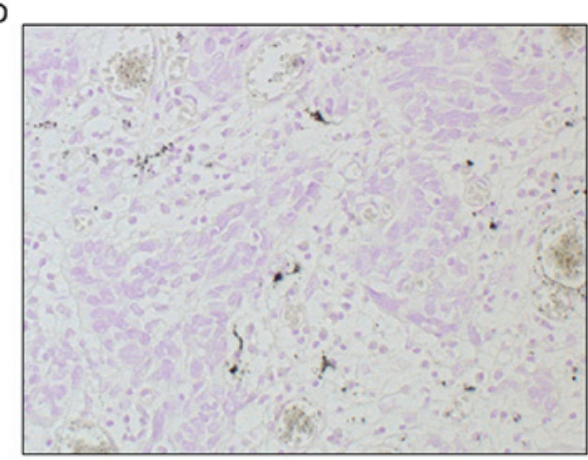

Figure 1. Representative cases of noninvasive low-grade papillary urothelial carcinoma (case no. 24) by (A) H\&E staining (magnification, x20) and (B) Feulgen reaction (magnification, $\mathrm{x} 40$ ); and infiltrating urothelial carcinoma (case no. 22) by (C) H\&E staining (magnification, x20) and (D) Feulgen reaction (magnification, $\mathrm{x} 40$ ). H\&E, hematoxylin and eosin.

$0.25 \mu \mathrm{m} / \mathrm{pixel}$; focus, autofocus; and source of lamination, super luminosity light emitting diode.

Image analysis. For Feulgen reaction images, representative $\mathrm{x} 40$ magnification digital images from three independent specimens were captured and saved as TIFF images, $\geq 100$ nuclei/sample were analyzed. Feulgen reaction stained nuclear areas were analyzed using Image-Pro Plus software (version 7.0.1.658; Japan Rover, Tokyo, Japan) The following parameters were obtained and used for statistical analysis: Average and standard deviation (SD) of size, density, maximum density, maximum diameter and minimum diameter of each separated Fuelgen reaction positive area. Subsequently, the mean and SD of these parameters were used for statistical analysis. DABand hematoxylin-stained nuclei from each tumor area were respectively counted using Analista software (version 1.0.7.4; Claro), and the positive ratio was determined.

Statistical analyses. For statistical analysis, JMP version 9.0.2 (SAS Institute Japan, Tokyo, Japan) was used. For comparison of two groups, if two groups were homoscedastic, Student's t-test (two sample t-test) was performed, if not, two sample t-test with Welch's correction was performed. For comparison of 3 groups, Kruskal-Wallis test was performed. The evaluated statistics were compared using $\chi^{2}$ distribution with the software and the probability was determined. For non-parametric multiple comparison, Steel-Dwass analysis was performed In all analyses, $\mathrm{P}<0.05$ was considered to indicate a statistically significant difference. The estimation of the area under the curve (AUC) value of a receiver operating characteristic (ROC) curve was performed as follows: An area $>0.9$ was considered to have high accuracy, whereas $>0.7$ and $\leq 0.9$ indicated moderate accuracy, $\geq 0.5$ and $\leq 0.7$ for low accuracy and $<0.5$ was a chance result (12). To determine the cutoff value of the ROC curve, the Youden index was used (13). In brief, the ROC table that was composed of each value with it's probability, including 1-specificity, sensitivity, sensitivity-(1-specificity), true positive, true negative, false positive and false negative, calculated by JMP version 9.0.2 software. The ROC curve was created by plotting each 1-specificity and sensitivity of the ROC table. The cutoff value was determined from the point on the ROC curve, which had greatest value of [sensitivity-(1-specificity)]. In order to determine the with the greatest value, the point on the ROC curve, which gave the longest perpendicular line from the diagonal line drawn from original point, was determined. The cutoff value was the value in the ROC table with this point.

\section{Results}

IUC and LGPUC are distinguished by nuclear parameters analyzed using IA. Since there was only one case (case no. 11) of high-grade non-infiltrative papillary urothelial carcinoma, the present study excluded this case from further analysis. Clear DNA ploidy pattern is obtained by Feulgen reaction in comparison to H\&E or Papanicolaou staining (14), thus the present study utilized Feulgen reaction for IA. First, the present study analyzed the nuclear area from tissue samples stained with Feulgen reagent. Representative staining of LGPUC and IUC tissue samples using H\&E, and Feulgen reagent are presented in Fig. 1. Following IA of Feulgen reaction specimens, the nuclear average density (average density of 

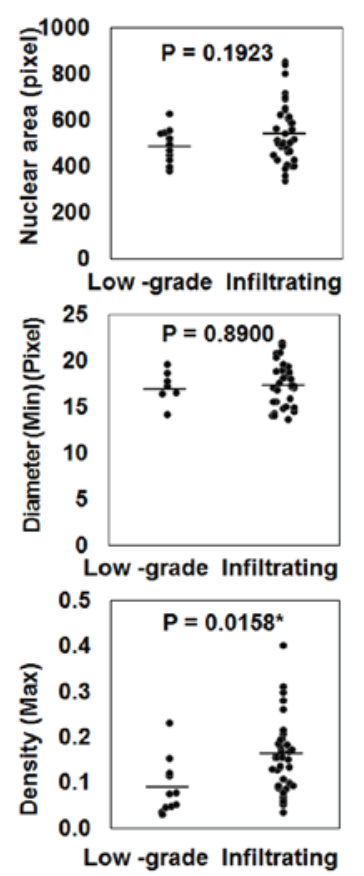
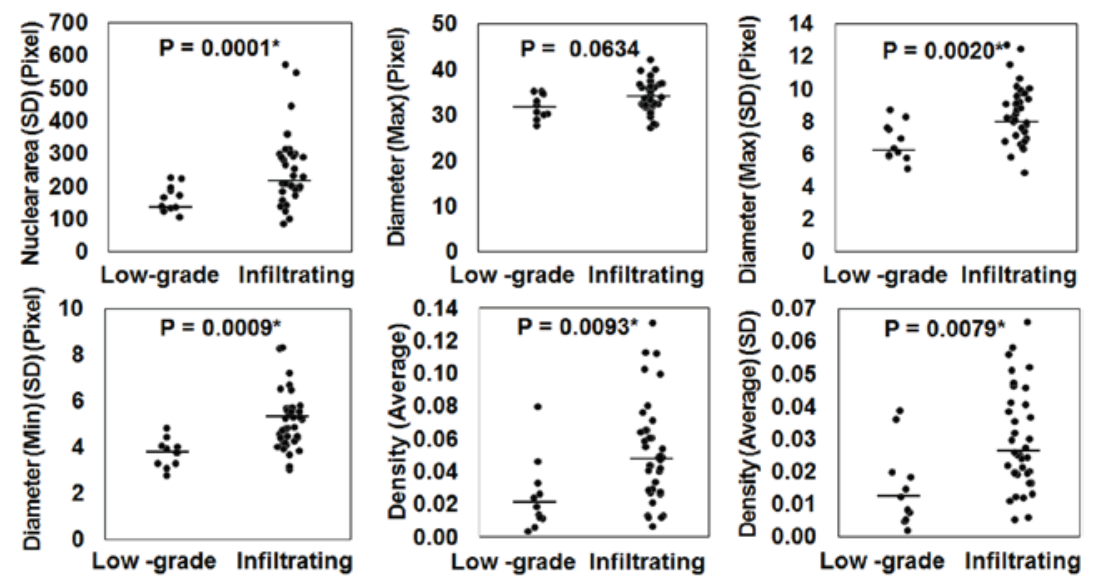

Figure 2. Scatter plot of nuclear parameters for Feulgen reaction tissue samples in low-grade papillary urothelial carcinoma (low-grade) and infiltrating urothelial carcinoma (infiltrating). For statistical analysis, SD of nuclear area was analyzed by Student's t-test with Welch's correction because the distribution was not homoscedastic, otherwise, the data were analyzed by Student's t-test only. Data is presented as the mean \pm standard deviation. SD, standard deviation.

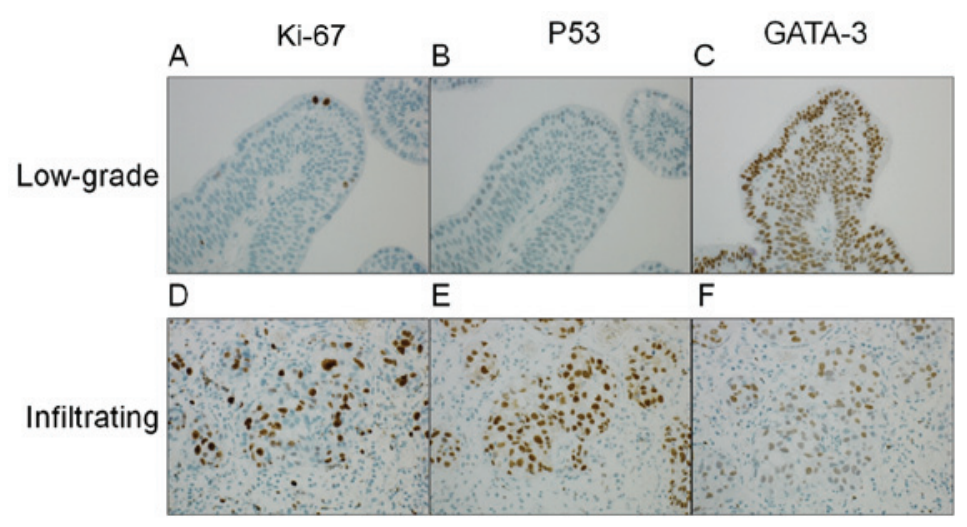

Figure 3. Representative immunohistochemical staining of GATA-3, Ki-67 and p53. Low-grade papillary urothelial carcinoma (case no. 24) stained for (A) Ki-67, (B) p53 and (C) GATA-3. Infiltrating urothelial carcinoma (case no. 22) stained for (D) Ki-67, (E) p53 and (F) GATA-3. GATA-3, GATA-binding protein 3. All images were captured using a x40 objective lens.

all pixels in a nucleus), nuclear maximum density (maximum density of pixels in a nucleus), SD of nuclear area, SD of nuclear maximum diameter, SD of nuclear minimum diameter, and SD of nuclear average density revealed statistically significant differences between IUC and LGPUC $(\mathrm{P}=0.0093$, $\mathrm{P}=0.0158, \mathrm{P}=0.0001, \mathrm{P}=0.0020, \mathrm{P}=0.0009$ and $\mathrm{P}=0.0079$, respectively; Fig. 2). However, the average nuclear area, average nuclear maximum diameter, average nuclear minimum diameter and SD of nuclear maximum density demonstrated no difference (Fig. 2). These data suggest that the average and the SD of each factor may be utilized to evaluate the nuclear characteristics of tumors.

Ki-67 expression is associated with carcinoma infiltration and GATA-3 downregulation was associated with muscular invasion. In the pathogenesis of urothelial carcinoma, p53 serves an important role for the development of IUC (15), whereas Ki-67 is associated with tumor grade and stage (16) and GATA-3 is downregulated during muscular invasion (17). The present study determined the expression levels of p53, Ki-67 and GATA-3 by IHC and analyzed the expression levels of each protein by IA. Representative staining patterns are presented in Fig. 3, and the positive ratio of Ki-67, p53 and GATA-3 stained cells in LGPUC and IUC were compared. As presented in Fig. 4, Ki-67, p53 and GATA-3 analysis of LGPUC and IUC tissues revealed significant differences between LGPUC and IUC tissue samples stained with Ki-67 $(\mathrm{P}<0.0001), \mathrm{p} 53(\mathrm{P}=0.0191)$ and GATA-3 $(\mathrm{P}=0.0087)$. Comparison of positive ratio of each protein among groups classified based on pathological $\mathrm{T}(\mathrm{pT})$ factor (pTa: papillary 

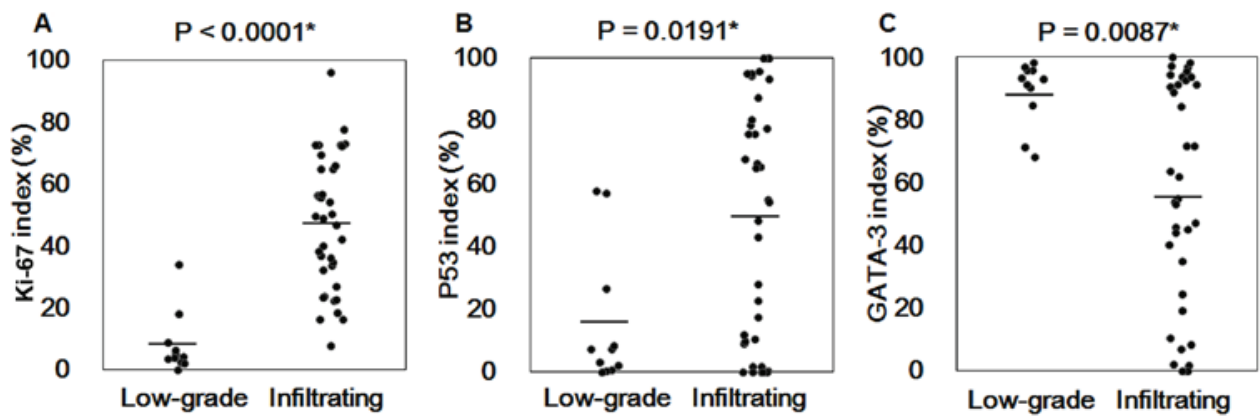

Figure 4. Scatter plot of positive ratios for (A) Ki-67, (B) p53 and (C) GATA-3 in tissue samples of low-grade papillary urothelial carcinoma (low-grade) and infiltrating urothelial carcinoma (infiltrating). For statistical analysis, the data were analyzed by Student's t-test. Data is presented as the mean \pm standard deviation. GATA-3, GATA-binding protein 3.

Table II. Association between pT factor and Ki-67, p53 or GATA-3 expression.

\begin{tabular}{llcccc}
\hline Index & pT & Number of cases & Rank sum & Expected value & Average of the ranks \\
\hline p53 & pTa & 15 & 284.5 & 375 & 18.97 \\
& pT1 & 13 & 425.0 & 325 & 32.69 \\
& pT2 & 21 & 515.5 & 525 & 24.55 \\
Ki-67 & pTa & 15 & 193.0 & 375 & 12.87 \\
& pT1 & 13 & 376.0 & 325 & 28.92 \\
\multirow{5}{*}{ GATA-3 } & pT2 & 21 & 656.0 & 525 & 31.24 \\
& pTa & 15 & 536.5 & 375 & 35.77 \\
& pT1 & 13 & 380.5 & 325 & 29.27 \\
\end{tabular}

Kurskal-Wallis test was performed. aStatistically significant. GATA-3, GATA-binding protein 3; pT, pathological T factor; pTa, papillary tumor without invasion; pT1, tumor with submucosal invasion; pT2, tumor with muscular invasion.

Table III. Non-parametric multiple comparison for Ki-67, p53 and GATA-3.

\begin{tabular}{llcc}
\hline Index & \multicolumn{2}{l}{ Compared groups } & P-value \\
\hline p53 & pTa & pT2 & 0.6713 \\
& pT2 & pT1 & 0.4084 \\
& pTa & pT1 & $0.0104^{\mathrm{a}}$ \\
Ki-67 & pT2 & pT1 & 0.8376 \\
& pTa & pT1 & $0.0057^{\mathrm{a}}$ \\
& pTa & pT2 & $0.0007^{\mathrm{a}}$ \\
GATA-3 & pTa & pT2 & $<0.0001^{\mathrm{a}}$ \\
& pTa & pT1 & 0.3382 \\
& pT2 & pT1 & $0.0065^{\mathrm{a}}$ \\
\hline
\end{tabular}

Steel-Dwass analysis was performed. 'Statistically significant. GATA-3, GATA-binding protein 3; pT, pathological T factor; pTa, papillary tumor without invasion; pT1, tumor with submucosal invasion; pT2, tumor with muscular invasion.

tumor without invasion; pT1: invasion up to submucosa; $\mathrm{pT2}$ : invasion to muscular layer) is summarized in Tables II and III. The expression levels of each protein were significantly different between 3 groups, as revealed by Kruskal Wallis test (p53 P=0.0394; Ki-67 P=0.0004 and GATA-3 P $<0.0001$, respectively; Table II). Therefore, additional statistical analysis was performed by a Steel-Dwass analysis for multiple comparisons of each pT factor. Ki-67 was significantly overexpressed in IUC cases, (pT1 and pT 2 cases, $\mathrm{P}=0.0057$ and $\mathrm{P}=0.0007$, respectively); however, p53 was significantly overexpressed in $\mathrm{pT} 1$ compared with pTa cases $(\mathrm{P}=0.0104)$, and $\mathrm{pT} 2$ cases demonstrated no significant differences compared with pTa or pT1 (Table III). Furthermore, GATA-3 expression level was significantly downregulated in $\mathrm{pT} 2$ cases $(\mathrm{P}<0.0001$ for $\mathrm{pTa}$ and $\mathrm{P}=0.0065$ for $\mathrm{pT} 1$ ). These results suggest that IUC may be distinguished from LGPUC by utilizing Ki-67 and p53, and muscle invasion cases may be identified from non-muscle invasion cases by GATA-3.

IUC and muscle invasion cases are predicted by cutoff values of Ki-67, p53 and GATA-3 positive ratios determined by image analysis. The present study aimed to determine the cutoff values of $\mathrm{Ki}-67, \mathrm{p} 53$ and GATA-3 positive ratios in order to distinguish IUC from LGPUC using Ki-67 and p53 or muscular invasion cases (pT2) from non-muscle invasion cases (pTa plus pT1), using GATA-3 and utilizing ROC curves and Youden index analyses. As presented in Fig. 5, the AUC for the Ki-67 index (LGPUC vs. IUC) was 0.96314 and the cutoff value was $18.7 \%$. The AUC for the p53 index (LGPUC vs. IUC) was 0.73587 and the cutoff value was $9.2 \%$. The AUC for the GATA-3 index (pT2 vs. pTa + pT1) was 0.86420 and the 
A

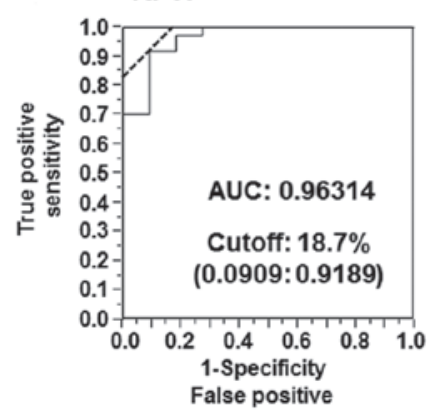

B

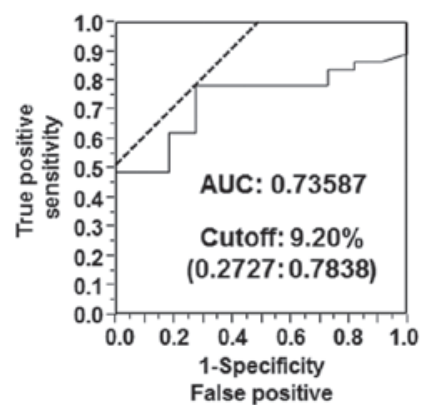

C

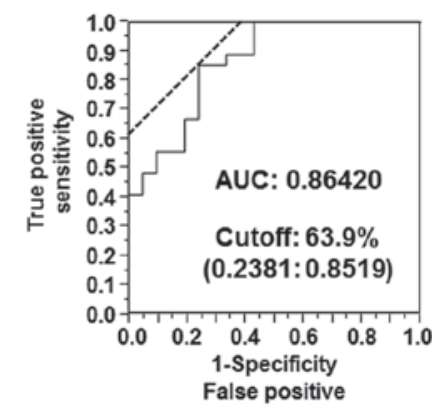

Figure 5. ROC curve of positive ratios for (A) Ki-67, (B) p53 and (C) GATA-3. AUC value in each graph was the ratio of the area under the curve to the total area of the graph, and the points beneath the cutoff value were the points on the ROC curve with the greatest value of [sensitivity-(1-specificity)]. The dotted line is a tangent line contacted on the point and gives the greatest value parallel to the diagonal line drawn from original point in the graph in order to demonstrate the point that is the most distant point from the diagonal line. GATA-3, GATA-binding protein 3; AUC area under the curve; LGPUC, low-grade papillary urothelial carcinoma; IUC, infiltrating urothelial carcinoma; pT, pathological T factor; pT2, muscular invasion cases; pTa + pT1, non-muscle invasion cases; ROC, receiver operating characteristic.

cutoff value was $63.9 \%$. Therefore, these results suggest that the combination of p53, Ki-67 and GATA-3 expression levels may be used to distinguish LGPUC cases from IUC cases and muscle invasion cases from non-muscle invasion cases.

\section{Discussion}

Previous studies using computer-aided cytopathological or histological IA emerged in the early 1980 s (18-21). At that time, the number of image elements were small [880x680 pixels (21) or $512 \times 625$ pixels (20)] and the software was too simple to clearly analyze nuclear morphology, or morphological structure in comparison to the software currently available (22). However, the advancement of technology has enabled us to analyze digital images captured by WSI, in which the analyses provide novel information for morphological diagnosis. For example, Caie et al (23) revealed that a poorly differentiated area detected by digital IA was associated with patient prognosis in colorectal cancer. Additionally, Ali et al (24) demonstrated that the median lymphocyte density of digital images from breast cancer biopsy specimen analyzed using IA software was associated with a pathological complete response to chemotherapy. The present study utilized WSI and IA to clarify nuclear features from urothelial carcinoma tissue samples as nuclear morphological analysis of urothelial carcinoma had not been previously performed in detail. However, certain studies have performed cell size analysis through digital imaging in bladder tissue samples (25), morphometric detection of urothelial cancer cell nests using an algorithm (5) and quantitative assessment of bladder carcinoma using an acid labile DNA assay (26). The results of the present study revealed that the SD of nuclear maximum and minimum diameter, SD of nuclear area (size), SD of nuclear average density, SD of nuclear maximum density, nuclear average density and nuclear maximum density were significantly higher in IUC compared with in LGPUC samples. However, the average nuclear area, average nuclear maximum diameter and average nuclear minimum diameter revealed no significant differences in IUC compared with LGPUC. SD is a measure of statistical variability of samples; therefore, SD was rephrased as nuclear variability in the present study. The nuclear findings of IUC have been previously described as "striking nuclear pleomorphism with variably sized and shaped hyperchromatic nuclei' (6). The present study confirmed that nuclear pleomorphism with variable size and shape by SD of nuclear size, SD of maximum nuclear diameter and SD of minimum nuclear diameter. Furthermore, hyperchromatic nuclei were identified by an average of nuclear density. In addition, the results of the present suggest that SD of nuclear density represents the nuclear variability of chromatin in each nucleus. Therefore, the term 'hyperchromatic nuclei' is not sufficient to express the status of a nucleus in IUC. The IA results indicated that the nuclei in IUC cases were globally hyperchromatic and variably hyperchromatic.

Krabbe et al (27) revealed that overexpression of Ki-67 using a $20 \%$ cutoff value was useful to predict recurrence-free survival and cancer-specific survival of patients with high-grade upper tract urothelial carcinoma. In the present study, the cutoff value determined by the Youden index of the ROC curve for Ki-67 was 18.7, with a highly accurate AUC value $(0.96314)$. The cutoff value determined by positive ratio detection using a combination of IA software and WSI was similar to the cutoff value used by Krabbe et al (27). Thus, the cutoff value used in the present study was reasonable, and IA in combination with WSI is a useful tool to evaluate IHC staining.

Biomarkers, including p53, have previously been intensively utilized for the investigation of urothelial carcinoma (28-30). By utilizing p53 IHC, Kalantari and Ahmadnia (31) reported positive rates of p53 in $75 \%$ of LGPUC samples and $85 \%$ of IUC samples by utilizing a $10 \%$ cutoff value. In the present study, the AUC for the p53 index (LGPUC vs. IUC) was 0.73587 (moderate accuracy) and the cutoff value was $9.2 \%$. By using this p53 cutoff value, 29/37 (78.4\%) IUC cases demonstrated overexpression of $\mathrm{p} 53$; thus, the cutoff value determined by IA was reasonable. However, according to a review article by Knowles and Hurst (15), the frequency of p53 overexpression is $30-50 \%$ in muscle invasion cases (15). Therefore, the estimation of p53 overexpression using a cutoff value is relatively variable. Consequently, careful consideration should be taken when interpreting p53 expression results following IHC. 
GATA family transcription factors have previously been investigated primarily in blood diseases (32). As a result, among the six GATA family factors (GATA-1 to -6), GATA-1, -2 and -3 have been termed 'hematopoietic GATA', and GATA- $4,-5$ and -6 termed 'endodermal GATA' (33). However, previous studies revealed that hematopoietic GATA member, GATA-3, is expressed in mammary glands $(33,34)$ and urothelium (35). As for GATA-3 expression in urothelial carcinoma, Miyamoto et al (17) reported that urothelial carcinoma with muscular invasion demonstrated downregulation of GATA-3 expression using a scoring system. The present study confirmed the downregulation of GATA-3 expression in pT2 IUC cases by positive rate calculated using IA software.

The present study determined the cutoff values of Ki-67 and p53 indexes in LGPUC, and IUC samples, or GATA-3 index between muscle invasion cases and non-muscle invasion cases. Our results indicated that IA may be useful for evaluation of IHC in an objective manner. Therefore, by utilizing WSI and IA software, reproducibility was superior to that of a scoring system to evaluate IHC results. Indeed, Papathomas et al (36) advocated that the current practices in the scoring assessment of Ki-67 varied greatly and that inter-observer variation set particular limitations to its clinical utility, particularly around clinically relevant cutoff values. It was concluded that novel digital microscopy-enabled methods may aid in reducing variation, increasing reproducibility and improving reliability in the clinical setting (36).

In conclusion, the present study revealed the usefulness of WSI and IA to evaluate nuclear morphological features and IHC results due to the objectivity of IA in comparison to manual evaluation, including scoring.

\section{Acknowledgements}

The authors would like to thank Dr Reika Takamatsu from the Department of Pathology and Oncology, Graduate School of Medicine, University of the Ryukus (Nishihara, Japan) for her expert technical assistance provided.

\section{References}

1. Pantanowitz L, Valenstein PN, Evans AJ, Kaplan KJ, Pfeifer JD, Wilbur DC, Collins LC and Colgan TJ: Review of the current state of whole slide imaging in pathology. J Pathol Inform 2: 36 , 2011.

2. Goacher E, Randell R, Williams B and Treanor D: The diagnostic concordance of whole slide imaging and light microscopy: A systematic review. Arch Pathol Lab Med 141: 151-161, 2017.

3. Calvaruso V, Burroughs AK, Standish R, Manousou P, Grillo F, Leandro G, Maimone S, Pleguezuelo M, Xirouchakis I, Guerrini GP, et al: Computer-assisted image analysis of liver collagen: Relationship to Ishak scoring and hepatic venous pressure gradient. Hepatology 49: 1236-1244, 2009.

4. Yamada M, Saito A, Yamamoto Y, Cosatto E, Kurata A, Nagao T, Tateishi A and Kuroda M: Quantitative nucleic features are effective for discrimination of intraductal proliferative lesions of the breast. J Pathol Inform 7: 1, 2016.

5. Hipp J, Smith SC, Cheng J, Tomlins SA, Monaco J, Madabhushi A, Kunju LP and Balis UJ: Optimization of complex cancer morphology detection using the SIVQ pattern recognition algorithm. Anal Cell Pathol (Amst) 35: 41-50, 2012.

6. Grignon DJ, Al-Ahmadie H, Algaba F, et al: Infiltrating urothelial carcinoma. In: WHO Classification of Tumors of the Urinary Systems and Male Genital Organs. Moch H, Humphrey PA Ulbright TM and Reuter VE (eds.) IARC, Lyon, pp81-98, 2016.
7. Reuter VE, Algaba F, Amin MB, et al: Non-invasive urothelial lesions. In: WHO Classification of Tumors of the Urinary Systems and Male Genital Organs. Moch H, Humphrey PA, Ulbright TM and Reuter VE (eds.) IARC, Lyon, pp99-107, 2016.

8. Loghavi S, Al-Ibraheemi A, Zuo Z, Garcia-Manero G, Yabe M, Wang SA, Kantarjian HM, Yin CC, Miranda RN, Luthra R, et al: TP53 overexpression is an independent adverse prognostic factor in de novo myelodysplastic syndromes with fibrosis. Br J Haematol 171: 91-99, 2015.

9. Fang JC, Xia ZX, Wang CN and Li Z: Clinicopathologic and immunophenotypic features of primary intestinal extranodal NK/T-Cell Lymphoma, Nasal Type. Int J Surg Pathol 23: 609-616, 2015.

10. Watanabe G, Ishida T, Furuta A, Takahashi S, Watanabe M, Nakata $\mathrm{H}$, Kato $\mathrm{S}$, Ishioka $\mathrm{C}$ and Ohuchi $\mathrm{N}$ : Combined immunohistochemistry of PLK1, p21, and p53 for predicting TP53 status: An independent prognostic factor of breast cancer. Am J Surg Pathol 39: 1026-1034, 2015.

11. Sobin LH, Gospodarowicz MK and Wittekind C: TNM Classification of Malignant Tumours. 7th edition. Wiley-Blackwell, Hoboken, NJ, 2009.

12. Akobeng AK: Understanding diagnostic tests 3: Receiver operating characteristic curves. Acta Paediatr 96: 644-647, 2007.

13. Perkins NJ and Schisterman EF: The inconsistency of 'optimal' cutpoints obtained using two criteria based on the receiver operating characteristic curve. Am J Epidemiol 163: 670-675, 2006.

14. Biesterfeld S, Beckers S, Del Carmen Villa Cadenas M and Schramm M: Feulgen staining remains the gold standard for precise DNA image cytometry. Anticancer Res 31: 53-58, 2011.

15. Knowles MA and Hurst CD: Molecular biology of bladder cancer: New insights into pathogenesis and clinical diversity. Nat Rev Cancer 15: 25-41, 2015.

16. Enache M, Simionescu C and Lascu LC: Ki67 and Bcl-2 immunoexpression in primitive urothelial bladder carcinoma. Rom J Morphol Embryol 53: 521-525, 2012.

17. Miyamoto H, Izumi K, Yao JL, Li Y, Yang Q, McMahon LA, Gonzalez-Roibon N, Hicks DG, Tacha D and Netto GJ: GATA binding protein 3 is down-regulated in bladder cancer yet strong expression is an independent predictor of poor prognosis in invasive tumor. Hum Pathol 43: 2033-2040, 2012

18. Rosenthal DL, McLatchie C, Stern E, White BS and Castleman KR: Endocervical columnar cell atypia coincident with cervical neoplasia characterized by digital image analysis. Acta Cytol 26: 115-120, 1982

19. Stern E, Rosenthal DL, McLatchie C, White BS and Castleman KR: An expanded cervical cell classification system validated by automated measurements. Anal Quant Cytol 4: 110-114, 1982.

20. Kriete A, Romen W, Schäffer R, Harms H, Haucke M, Gerlach B, Aus HM and ter Meulen V: Computer analysis of chromatin arrangement and nuclear texture in follicular thyroid tumours. Histochemistry 78: 227-230, 1983.

21. Komitowski D and Zinser G: Quantitative description of chromatin structure during neoplasia by the method of image processing. Anal Quant Cytol Histol 7: 178-182, 1985.

22. Saito A, Numata Y, Hamada T, Horisawa T, Cosatto E, Graf HP, Kuroda $\mathrm{M}$ and Yamamoto Y: A novel method for morphological pleomorphism and heterogeneity quantitative measurement: Named cell feature level co-occurrence matrix. J Pathol Inform 7: $36,2016$.

23. Caie PD, Zhou Y, Turnbull AK, Oniscu A and Harrison DJ: Novel histopathologic feature identified through image analysis augments stage II colorectal cancer clinical reporting. Oncotarget 7: 44381-44394, 2016.

24. Ali HR, Dariush A, Provenzano E, Bardwell H, Abraham JE, Iddawela M, Vallier AL, Hiller L, Dunn JA, Bowden SJ, et al: Computational pathology of pre-treatment biopsies identifies lymphocyte density as a predictor of response to neoadjuvant chemotherapy in breast cancer. Breast Cancer Res 18: 21, 2016.

25. Keshtkar A, Keshtkar A and Lawford P: Cellular morphological parameters of the human urinary bladder (malignant and normal). Int J Exp Pathol 88: 185-190, 2007.

26. Gschwendtner A and Mairinger T: Quantitative assessment of bladder carcinoma by acid labile DNA assay. Cancer 86: 105-113, 1999.

27. Krabbe LM, Bagrodia A, Lotan Y, Gayed BA, Darwish OM, Youssef RF, John G, Harrow B, Jacobs C, Gaitonde M, et al: Prospective analysis of $\mathrm{Ki}-67$ as an independent predictor of oncologic outcomes in patients with high grade upper tract urothelial carcinoma. J Urol 191: 28-34, 2014 
28. Shariat SF, Karakiewicz PI, Ashfaq R, Lerner SP, Palapattu GS, Cote RJ, Sagalowsky AI and Lotan Y: Multiple biomarkers improve prediction of bladder cancer recurrence and mortality in patients undergoing cystectomy. Cancer 112: 315-325, 2008.

29. Shariat SF, Chade DC, Karakiewicz PI, Ashfaq R, Isbarn H Fradet Y, Bastian PJ, Nielsen ME, Capitanio U, Jeldres C, et al: Combination of multiple molecular markers can improve prognostication in patients with locally advanced and lymph node positive bladder cancer. J Urol 183: 68-75, 2010.

30. Shariat SF, Ashfaq R, Sagalowsky AI and Lotan Y: Predictive value of cell cycle biomarkers in nonmuscle invasive bladder transitional cell carcinoma. J Urol 177: 481-487, 2007.

31. Kalantari MR and Ahmadnia H: P53 overexpression in bladder urothelial neoplasms: New aspect of World Health Organization/International Society of Urological Pathology classification. Urol J 4: 230-233, 2007.

32. Gao J, Chen YH and Peterson LC: GATA family transcriptional factors: Emerging suspects in hematologic disorders. Exp Hematol Oncol 4: 28, 2015.
33. Zheng R and Blobel GA: GATA transcription factors and cancer. Genes Cancer 1: 1178-1188, 2010.

34. Asselin-Labat ML, Sutherland KD, Barker H, Thomas R, Shackleton M, Forrest NC, Hartley L, Robb L, Grosveld FG, van der Wees J, et al: Gata-3 is an essential regulator of mammary-gland morphogenesis and luminal-cell differentiation. Nat Cell Biol 9: 201-209, 2007.

35. Liang Y, Heitzman J, Kamat AM, Dinney CP, Czerniak B and Guo CC: Differential expression of GATA-3 in urothelial carcinoma variants. Hum Pathol 45: 1466-1472, 2014.

36. Papathomas TG, Pucci E, Giordano TJ, Lu H, Duregon E, Volante M, Papotti M, Lloyd RV, Tischler AS, van Nederveen FH, et al: An international Ki67 reproducibility study in adrenal cortical carcinoma. Am J Surg Pathol 40: 569-576, 2016. 